\title{
COMMENTARY
}

\section{Dimebon disappointment}

Roy W Jones*

\begin{abstract}
Dimebon (latrepirdine) has received widespread publicity as a potential therapy for Alzheimer's disease following a very positive phase 2 study carried out in Russia and published in the Lancet in 2008. In this study there were improvements over 6 months in all endpoints (cognitive, global, daily function and behaviour), with continuing improvement at 12 months in cognition and daily function. A more recent multinational phase 3 study, however, showed no improvements whatsoever and no difference between the two drug-treated groups and the placebo group. Of note, there was little deterioration in any of the groups after 6 months in contrast to the placebo group in the phase 2 study. The potential reasons for these disappointing results are discussed, as well as the implication for dimebon and drug treatment in Alzheimer's disease.
\end{abstract}

One swallow does not make a summer, and one positive clinical trial does not make an Alzheimer's drug. This was the Alzheimer Research Forum's response in March 2010 [1] to the news release by Pfizer Inc. and Medivation Inc. of the much-awaited data from the phase 3 CONNECTION study with dimebon (latrepirdine) [2]. Unfortunately, the trial met neither its co-primary (cognition and global function) nor its secondary efficacy endpoints. This disappointing news increased scepticism about the unusually positive results of the original phase 2 trial carried out in Russia and published in the Lancet in 2008 [3].

Dimebon is orally available and was previously approved in Russia as a nonselective antihistamine but withdrawn from the market with the development of more selective compounds [4]. More recent papers described weak inhibition of butyrylcholinesterase, acetylcholinesterase, the $N$-methyl-D-aspartate receptor

*Correspondence: R.W.Jones@bath.ac.uk

Research Institute for the Care of Older People, Royal United Hospital, Bath BA1 3NG, UK signalling pathway and the mitochondrial permeability transition pore opening [4-7]. Together with the demonstration of neuroprotective effects in Alzheimer's disease (AD) and Huntington's disease models, these observations supported the potential of dimebon as a treatment for $\mathrm{AD}$ - although the plausibility of dimebon's mechanism of action has been queried [8].

In the phase 2 placebo-controlled study in mild-tomoderate AD funded by Medivation Inc., 155 patients (85\% of those enrolled) completed the study [3]. Dimebon (20 mg three times daily) was safe and well tolerated, and significantly improved the clinical course of patients [3]; the mean change from baseline scores significantly favoured the drug for all five outcome measures: two measures of cognition (Mini-mental State Examination, and Alzheimer's Disease Assessment Scale cognitive subscale), one measure of activities of daily living (Alzheimer's Disease Cooperative Study activities of daily living), one measure of behaviour (Neuropsychiatric Inventory) and a global rating scale (Clinician's Interviewbased Impression of Change plus Caregiver Input). The drug-placebo differences were not driven by worsening in the placebo group and there was a widening drugplacebo difference over the 26-week study. A 26-week blinded extension phase was included, and the Alzheimer's Disease Assessment Scale cognitive subscale and the Alzheimer's Disease Cooperative Study activities of daily living differences increased further from 26 to 52 weeks [3]. The authors commented that the increasing benefit was especially important because 'at present no approved therapies for mild-to-moderate AD have shown increasing improvement over 12 months' [3]; furthermore, other treatments had not shown such consistent results across several standardised measures within one study.

Not surprisingly, these results created a great deal of excitement and expectation - although the authors cautioned that this was a single-country trial of one dose of dimebon, and that a larger multinational study was needed to confirm the findings.

The CONNECTION study was the larger study cosponsored by Pfizer Inc. (who had now entered into a global collaboration with Medivation Inc. for the development of dimebon) involving 598 patients with mild-to-moderate $\mathrm{AD}$ at 63 sites in North America, 
Europe and South America [2]. Patients were randomised to one of three treatment groups, receiving either dimebon $20 \mathrm{mg}$ three times daily (as for the original study) or $5 \mathrm{mg}$ three times daily (to help define the effective dose range [2]) or placebo. The co-primary endpoints were cognition (Alzheimer's Disease Assessment Scale cognitive subscale) and global function (Clinician's Interview-based Impression of Change plus Caregiver Input). After 6 months of treatment, there were no significant differences between any of the three groups. Secondary endpoints were also negative, including the Mini-mental State Examination - in which the placebo group actually performed numerically better, although the differences were nonsignificant.

Dimebon was well tolerated in the CONNECTION study and also in a separate phase 3 safety and tolerability study, which confirmed dimebon's good tolerability when dosed alone or in combination with other approved AD medications [2].

So why are the study results so different? The authors of the phase 2 study comment that the patients were younger (mean age 68.1 years) than those usually recruited to Alzheimer's studies worldwide, and in the phase 3 trial the mean age was 74.4 years. In the Russian study, the mean baseline Mini-mental State Examination score was 18.7 compared with a mean score of 17.7 on entry to the phase 3 study. The benefits of dimebon in the phase 2 study were not driven by worsening in the placebo group, but reflected an actual improvement on dimebon as well as a decline on placebo. In the phase 3 trial, however, the patients in the trial did not deteriorate significantly in either the drugtreated group or the placebo group, which makes interpretation of the study more difficult. Patients in one country (Russia) might well be different from those taking part in a multinational study (in the phase 3 trial, more than $40 \%$ of the patients were from the USA).

Most comments seem to have questioned the validity of the Russian data, and there are potential concerns about this validity - as there have been about Russian data on an anticancer drug (NOV-002), which also showed dramatically positive results not supported by the subsequent phase 3 study [8]. The dimebon formulation used in Russia appears different from that of the multinational study. The original medication has a bitter taste and a numbing effect on the tongue [8], which could lead to unblinding, whereas in the later study the tablet was film-coated. There have been other comments about whether the same compound was used in both studies because of the different side-effect profiles [1]. Dry mouth was the commonest side effect seen in the Russian study (14\% of patients at 26 weeks and $18 \%$ at 52 weeks in comparison with just $1 \%$ on placebo) [3], but somnolence and headache were the commonest side effects in the multinational study (and the incidence was more balanced between drug-treated and placebo groups: somnolence, $11 \%$ active versus $10.1 \%$ placebo; headache, $9.5 \%$ versus $5.6 \%$; and dry mouth, $8.5 \%$ versus $6.6 \%)[2]$.

Equally questionable is whether the multinational study recruited typical patients, especially when more than $40 \%$ were recruited from the USA. The study did not allow recruitment of patients who were receiving or had received within 90 days either cholinesterase inhibitors or memantine. The normal treatment for most patients with AD would be a cholinesterase inhibitor or memantine or combination therapy, and it may therefore be that patients who do not receive this are atypical yet they would be the patients recruited to the study. There are clearly a number of other differences between the Russian phase 2 study and the multinational phase 3 study.

The results of the phase 3 study have led to further negative comments about the underlying rationale for the use of dimebon in $\mathrm{AD}$ and the limited preclinical data that are available. Dr Samuel Gandy commented that 'this was a drug with no plausible mechanism that emerged from an incomprehensible series of screens', whilst Dr Lon Schneider commented on the limited pharmacokinetic data available [8].

The negative phase 3 study has already led to a reduction in the dimebon clinical trial programme, with a number of studies having stopped (for example, see study NCT01066546 on clinicaltrials.gov). One 12-month study (NCT00829374) is continuing, however, comparing two doses of dimebon with placebo and recruiting 1,050 patients with mild-to-moderate $\mathrm{AD}$ who are stable on donepezil. These patients are likely to be more typical than those in the previous phase 3 monotherapy study and are being recruited in the USA, Europe, Australia and New Zealand; it is important that we wait for these results. If the results of this study are also negative, then yet another potential Alzheimer's therapy will have fallen at the phase 3 hurdle - although in this case after a strong phase 2 signal that perhaps was too good to be true. This will be disappointing and would confirm how far we may still have to go before we achieve real advances in therapy for patients with AD.

\section{Abbreviations}

$A D$, Alzheimer's disease.

\section{Competing Interests}

RWJ has received honoraria and consultancy fees from a number of companies involved in research and development of new drugs for

Alzheimer's disease, including Medivation and Pfizer, and his research institute has received grants in association with clinical trials in this area, including trials involving dimebon.

Published: 13 September 2010

\section{References}

1. Dimebon Disappoints in Phase 3 Trial [http://www.alzforum.org/new/detail. asp?id=2387] 
2. Pfizer and Medivation Announce Results from two Phase 3 Studies in Dimebon (Latrepirdine) Alzheimer's Disease Clinical Development

Program [http://investors.medivation.com/releasedetail. cfm?ReleaselD $=448818]$

3. Doody RS, Gavrilova SI, Sano M, Thomas RG, Aisen PS, Bachurin SO, Seely L, Hung $D$, on behalf of the dimebon investigators: Effect of dimebon on cognition, activities of daily living, behaviour, and global function in patients with mild-to-moderate Alzheimer's disease: a randomised, double-blind, placebo-controlled study. Lancet 2008, 372:207-215.

4. Bachurin S, Bukatina E, Lermontova N, Tkachenko S, Afanasiev A, Grigoriev V, Grigorieva I, Ivanov Y, Sablin S, Zefirov N: Antihistamine agent dimebon as a novel neuroprotector and a cognition enhancer. Ann NY Acad Sci 2001, 939:425-435.

5. Lermontova NN, Redkozubov AE, Shevtsova EF, Serkova TP, Kireeva EG, Bachurin SO: Dimebon and tacrine inhibit neurotoxic action of betaamyloid in culture and block L-type $\mathrm{Ca}^{2+}$ channels. Bull Exp Biol Med 2001, 132:1079-1083
6. Grigor'ev W, Dranyi OA, Bachurin SO: Comparative study of action mechanisms of dimebon and memantine on AMPA- and NMDA-subtypes glutamate receptors in rat cerebral neurons. Bull Exp Biol Med 2003, 136:474-477.

7. Bachurin SO, Shevtsova EP, Kireeva EG, Oxenkrug GF, Sablin SO: Mitochondria as a target for neurotoxins and neuroprotective agents. Ann NY Acad Sci 2003, 993:334-344.

8. Jeffrey S: Dimebon disappoints: is there hope for novel Alzheimer's agent? Medscape Medical News [www.Google.com: Medscape Dimebon].

doi:10.1186/alzrt49

Cite this article as: Jones RW: Dimebon disappointment. Alzheimer's Research \& Therapy 2010, 2:25. 